\title{
Rosacea infant: not always a benign dermatosis
}

\author{
Sabah Bazouti', Loubab Omahsan', Hanane Chahib², Rachid Sakhsoukh², \\ Siham Dikhaye ${ }^{1,3}$, Nada Zizi ${ }^{1,3}$
}

${ }^{1}$ Department of Dermatology, Mohammed VI University Hospital of Oujda, Medical School of Oujda, Mohammed First University of Oujda, Oujda, Morocco, ${ }^{2}$ Department of Ophthalmology, Mohammed VI University Hospital of Oujda, Medical School of Oujda, Mohammed First University of Oujda, Oujda, Morocco, ${ }^{3}$ Epidemiology Laboratory of Research and Public

Health, Oujda, Morocco

Corresponding author: Dr. Sabah Bazouti, E-mail: Sabah.bazouti@gmail.com

\begin{abstract}
Rosacea is a condition that is most often associated with adults; however, various forms exist in the pediatric population and must be taken into account when a child presents a rash to the face. We report a rare case of rosacea with severe ocular involvement in a 7-year-old child with discrete papules and pustules on both cheeks and chin with blepharitis and decreased visual acuity. The ophthalmologic examination confirmed a severe blepharitis with severe mibomitis associated with a stromal corneal ulcer. The diagnosis of rosacea with ocular involvement was made. Patient was put on oral azithromycin, topical metronidazole at the face and a photoprotection with good evolution. Early recognition and treatment of ocular rosacea in children can improve patient outcomes by limiting the progression of corneal pathology.
\end{abstract}

Key words: Pediatric rosacea; Ocular rosacea; Cutaneous rosacea

\section{INTRODUCTION}

Rosacea is a chronic inflammatory facial dermatitis. It mainly affects persons of middle age and a clear phototype, and it is considered rare in children [1]. Ocular involvement is the most serious complication requiring early diagnosis in order to preserve the visual function. We report a new case of ocular rosacea in a 7-year-old child.

\section{CASE REPORT}

A 7-year-old boy with a phototype III, and a family history of rosacea affecting his mother and maternal aunts, consulted the ophthalmic emergencies for a painful red right eye with significant photophobia. In the anamnesis, we find notion of flushes during temperature changes and repeated chalazion with a many similar episodes during the last two years wrongly treated as allergic conjunctivitis. Ophthalmological examination showed blepharitis with severe meibomitis associated with a stromal corneal ulcer (Fig. 1). A dermatological examination had objectified the presence of some erythematous papules, telangiectasias on a pink background of cheeks, chin and the palpébral edges (Fig. 2). The dermoscopic examination had shown the presence of arborizing vessels, sometimes polygonal, as well as pustules and erythematous background. The diagnosis of rosacea was retained and the patient was put on oral azitromycin, topical metronidazole in the face and photoprotection with a good evolution.

\section{DISCUSSION}

Rosacea is a fairly common chronic facial dermatitis, whose infantile forms are rare and often underdiagnosed. The cutaneous manifestations described in children, generally inconspicuous, seem identical to those observed in adults [2-3], with the exception of hypertrophic rosacea never reported in this age group [2]. Familial history of rosacea is often reported [1] as in our case which could support the diagnosis. Ocular signs, however, are not specific and can reach all levels of severity ranging from simple photophobia to blepharitis-meibomitis to blindness through a

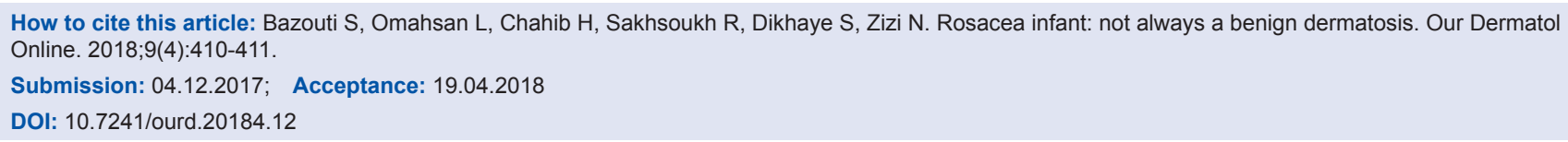




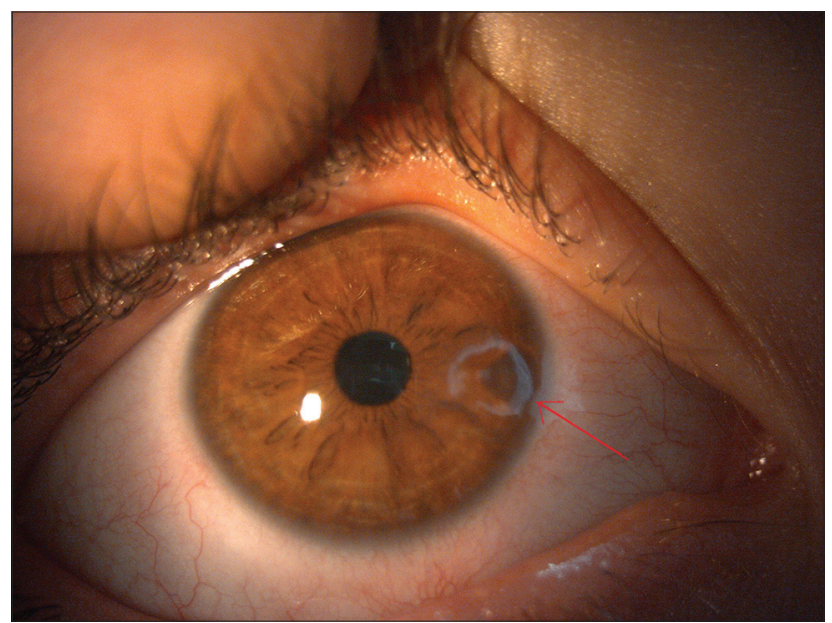

Figure 1: Clinical image showing a corneal ulcer.

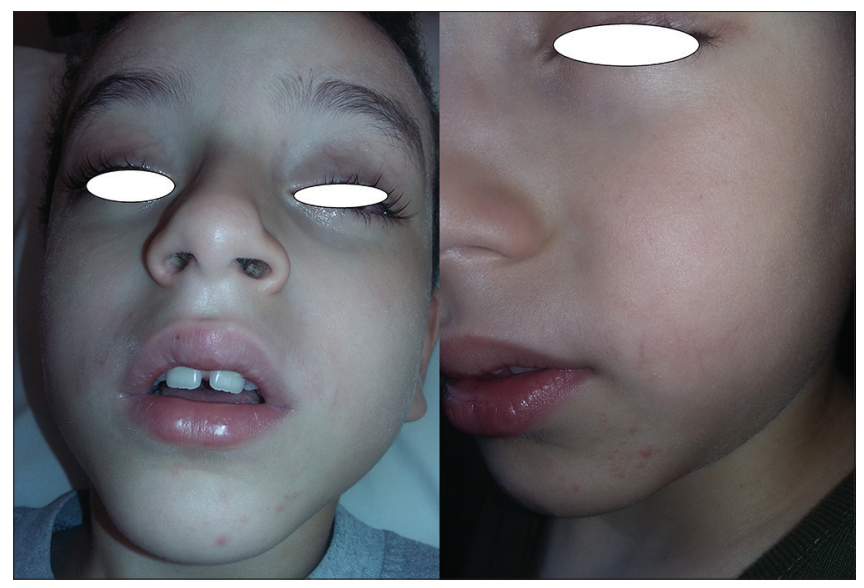

Figure 2: Papulopustular lesions associated with telangiectasia.

keratoconjunctivitis complicated sometimes by corneal ulcers [4] as was the case in our patient, imposing immediate care. There is no correlation between the severity of ocular rosacea and inflammatory cutaneous rosacea [5]. Although rosacea in children especially before puberty, is uncommon, ocular symptoms are often present at diagnosis [6].

The local treatment is identical to that of adult rosacea [1]. A systemic treatment is proposed in case of failure of topics or in case of severe ocular involvement. Systemic antibiotics, primarily the tetracyclines, are the drugs of choice for most individuals with rosacea. Tetracyclines should not be used in children younger than 8 years, because they can lead to discoloration of teeth and susceptibility to fracture by incorporation into bones and teeth. In this younger age group, macrolides are the preferred agents. Azithromycin can produce marked improvement, even when administered for only 5 days [7].

\section{CONCLUSION}

Infantile rosacea is a rare and potentially serious disease in its ocular form. Its presentation is not specific and can be confusing, especially in the absence of dermatological lesions. In front of a child with ocular signs suspicious of rosacea, the dermatological examination is an important step for making the diagnosis. Early management avoids serious complications such as keratitis and/or corneal ulcer. Only a multidisciplinary and effective management by dermatologist and ophthalmologist avoids the complications that can lead to blindness.

\section{CONSENT}

The examination of the patient was conducted according to the Declaration of Helsinki principles.

\section{REFERENCES}

1. Kroshinsky \& Glick. Pediatric rosacea. Dermatol Ther. 2006;19:196-201.

2. Wilkin J, Dahl M, Detmar M, Drake L, Feinstein A, Odom R, Powell F. Standard classification of rosacea: report of the National Rosacea Society Expert Committe on the classification and staging of Rosacea. J Am Acad Dermatol. 2002;46:584-7.

3. Kaveh GN, Rana R, Zeinab AS, Behnam R. Miliarial type pseudolymphoma mimicking as granulomatous rosacea. Our Dermatol Online. 2017;8:318-21.

4. Chamaillard M, Mortemousque B, Boralevi F, Marques da Costa C, Aitali F, Taïeb A, et al. Cutaneous and ocular signs of childhood rosacea. Arch Dermatol. 2008;144:167-71.

5. Keshtcar-Jafari A, Akhyani M, Ehsani AH, Ghiasi M, Lajevardi V, Baradran O, et al. Correlation of the severity of cutaneous rosacea with ocular rosacea. Indian J Dermatol Venereol Leprol 2009;75:405-6.

6. Potz-Biedermann C, Mehra T, Deuter C, Zierhut M, Schaller M. Ophthalmic Rosacea: Case Report in a Child and Treatment Recommendations. Pediatr Dermatol. 2015;32:522-5.

7. Donaldson KE, Karp CL, Dunbar MT. Evaluation and treatment of children with ocular rosacea. Cornea. 2007;26:42-6.

Copyright by Sabah Bazouti, et al. This is an open access article distributed under the terms of the Creative Commons Attribution License, which permits unrestricted use, distribution, and reproduction in any medium, provided the original author and source are credited. Source of Support: Nil, Conflict of Interest: None declared. 